\title{
A Comparison of Internal, External, and Successive Unfolding Based on Occupational Judgments
}

\author{
Michaอ T. Branmick and June Hahn \\ Bomling Green State University
}

\begin{abstract}
Successive unfolding is a recently developed technique that appears to have advantages over traditional unfolding techniques. To assess its usefulness, successive, internal, and a type of external unfolding were compared with regard to the stimulus configurations recovered, the fit of the models as a whole, the fit of individual subjects, and each model's stability in a cross-validation sample. The data were obtained from judgments of similarity of and preference for occupational titles. Internal unfolding yielded degenerate solutions and was dropped from subsequent analyses. External and successive unfolding yielded interpretable dimensions, but the nature of the dimensions uncovered differed somewhat from one type of unfolding to the other. Both types of unfolding fit the data adequately for the stimulus configuration, though successive unfolding appeared to fit better. The first two dimensions of both models were well reproduced in cross-validation samples. Dimensions beyond the second were more easily cross-validated using external unfolding. Successive unfolding proved superior to external unfolding in representing preference data as indicated by the fit of ideal points. Successive unfolding appears a viable method in unfolding research.
\end{abstract}

Methods for deriving joint spatial representations of individuals and objects have been available since Coombs' (1950) technique effectively "unfolded" preference rankings into a joint scale containing both persons and objects. An individual's

APPLIED PSYCHOLOGICAL MEASUREMENT

Vol. 9, No. 2, June 1985, pp. 199-208

(C) Copyright 1985 Applied Psychological Measurement Inc. 0146-6216/85/020199-10\$1.75 preferences corresponded to the rank order of the distances from that individual to the objects. Thus, the most preferred objects should be the closest to the individual. Coombs' original model assumed a single underlying dimension, and served both as method and criterion, that is, a set of data would or would not unfold. Bennett and Hays (1960) extended unfolding into multiple dimensions, and Carroll (1972) developed a hierarchy of multidimensional unfolding models relating level of preference to various functions of distance. Several recent books on multidimensional scaling (MDS) treat unfolding models in some detail (Coxon, 1982; Davison, 1983; Kruskal \& Wish, 1978; Schiffman, Reynolds, \& Young, 1981). There has been progress on metric unfolding as well (Davison, 1976 , 1977).

Unfolding analyses are commonly classified as either internal or external. Internal unfolding analyses provide estimates of stimuli coordinates and person coordinates using only raw preference data (usually ranks). It should be noted, however, that internal unfolding analyses frequently yield degenerate solutions due to computational problems (Schiffman et al., 1981). Consequently, other procedures have been developed, the most common called external unfolding. A stimulus space is obtained based on paired comparison similarity judgments or on some other matrix of similarities or dissimilarities. Preferences are subsequently mapped into this derived space (see, e.g., Carroll, 1972; Coxon \& Jones, 1979b; Schiffman et al., 1981). 
A more recent development is the technique of successive unfolding (Rogers \& Young, 1981). The only data required for this type of analysis are rank orderings. Successive unfolding involves four steps: (1) computing a matrix of interstimulus proximities from the preference orderings, (2) using ALSCAL metrically (Takane, Young, \& deLeeuw, 1977) to solve for the stimulus configuration, (3) using Carroll's (1972) regression procedure to estimate ideal points, and (4) nonmetrically placing the ideal points in the derived stimulus space. The result of such a procedure is a complete unfolding analysis. Both stimuli and people are represented in a common Euclidean space.

Successive unfolding is essentially a hybrid of internal and external unfolding. It is internal in that only preference data enter the analysis, and it is external in that a stimulus configuration is computed first, and ideal points are subsequently mapped into this space. Alternatively, successive unfolding may be considered a special case of external unfolding. The term "successive unfolding" is used here to distinguish between it and other forms of external unfolding.

Successive unfolding has advantages over both internal and most forms of external unfolding. Unlike internal unfolding, successive unfolding does not appear to be subject to the common form of degenerate solutions (see Carroll \& Arabie, 1980; Davison, 1983; Schiffman et al., 1981). Unlike external unfolding, successive unfolding involves only rank order preference judgments, saving both time and effort on the part of researchers and subjects. Because successive unfolding has these advantages over traditional unfolding methods, its application to new content areas appears worthwhile. However, a number of methodological questions need to be addressed prior to its use.

The first question involves the stability of successive unfolding solutions. How well does a stimulus space derived from one sample hold up when applied to a new sample? This question applies equally to internal, external, and successive unfolding, since each method involves least squares procedures which capitalize on chance. This important question is rarely addressed in published applications of MDS.
A second question is whether the spatial contiguration derived by successive unfolding differs substantially from spatial configurations derived by the more traditional methods of internal and externall unfolding. This is a key point, since one purpose of exploratory MDS is to uncover the dimensions or attributes that people use to make judgments about stimuli. Most external unfolding configurations are based on similarity data, whereas successive unfolding uses only preference data. Kruskal and Wish (1978) noted (1) that preference and similarity tasks may invoke different psychological processes and (2) that dimensions of similarity may be irrelevant to preference judgments. For example, hair color may be a dimension of discrimination in similarity judgments about the opposite sex, but irrelevant in dating preferences. Although little research has addressed the issue of preference and similarity judgments with regard to common cognitive maps, Sjöberg (1980) presented some arguments and data which suggested that at least a rough correspondence exists between MDS results based on similarity and preference judgments. He argued that a common cognitive map or mental structure is used by subjects for making both similarity and preference judgments. Nygren and Jones (1977) also compared spaces of political perception and preference; they concluded that essentially the same multidimensional representation of stimuli came from similarity and preference judgments.

Multidimensional unfolding (MDU) models may further be compared on the basis of how well they represent individuals. Since the object of MDU is to represent people's preferences geometrically, different MDU models may be compared as to the fit of ideal points. Because of computational procedures, both successive and internal unfolding can be expected to fit ideal points better than external unfolding. Preference information is used in deriving the stimulus spaces for both successive and internal unfolding.

To contrast the performance of the three MDU models, it is necessary to analyze a single set of data with each technique. Comparisons can then be made of the stimulus configurations recovered, the fit of the model as a whole, the fit of individual 
subjects, and each model's stability in a cross-validation sample.

Such comparisons were undertaken in the present study. The data used were derived from judgments about occupations. Occupations differ in many ways, offering complex bases for discrimination, categorization, and preference. The complexity of the stimuli in this study was intended to be representative of the type of stimuli other users of MDU might encounter. The stimuli used in developing external and successive unfolding have usually been considerably less complex than that of occupations. For example, in developing successive unfolding, Rogers and Young (1981) used family types as stimuli. These families differed only in the number, sex, and sex ratio of the chil dren. In developing external unfolding, Carroll (1972) analyzed preference and similarity judgments about hypothetical cups of tea that differed only in sugar content and temperature. In both cases the dimensions of the spatial configuration were effectively defined by the choice of stimuli. Although there is some support for the congruence of similarity and preference spaces with more complex datasets (Nygren \& Jones, 1977; Reeb, 1979; Sjoberg, 1980), the degree of congruence is likely to depend on the kind of stimuli, subjects, and tasks used in the study.

In summary, the purpose of the present study was (1) to uncover and label dimensions of occupational preference using internal, successive, and external unfolding to determine whether the models recover similar stimulus configurations; (2) to report the stability of the stimulus configuration obtained using each model, and to compare stability across unfolding models; and (3) to compare the fit of ideal points across unfolding models.

\section{Method}

\section{Surigects}

Subjects were 94 undergraduate volunteers who performed in groups ranging in size from 1 to 10 . Each subject received one hour of experimental credit in an introductory psychology course.

\section{Procedure}

Each subject performed three tasks: (1) completely ranking job titles by preference, (2) sorting job titles into categories on the basis of similarity, and (3) rating each job on four attributes. The preference ranks were used for internal unfolding and to generate the stimulus configuration for successive unfolding. The ratings of jobs were used to help interpret the scaling solutions. To control for order effects, groups of subjects were run with the ranking task first, or with the categorization task first. The schedule of orders was random. The rating task was always administered last, because it could have influenced the categorization and ranking responses, thereby "creating" dimensions.

Job ranks. Subjects ranked 25 job titles in order of preference. The job titles were randomly selected from a scale of occupational prestige (Hodge, Siegel, \& Rossi, 1968). Job titles were printed on index cards. Each subject shuffled the cards before ranking, so that different subjects viewed the job titles in different orders.

Categorization. Subjects categorized jobs into similar groups using the free categorization task presented by Rosenberg, Nelson, and Vivekananthan (1968). Subjects sorted job titles, which somehow "went together," into pilles, the number and nature of the piles being determined by each subject. Proximities were obtained by counting the number of subjects who placed two stimuli into the same category. The co-occurrence frequencies were subtracted from the number of subjects to create a dissimilarity matrix. Diagonals were set to zero. This procedure was chosen because the large number of stimuli precluded presenting all pairwise combinations, and deleting pairs results in information loss that may lead to spatial distortion (Golledge \& Rayner, 1982). Further, since sorting has been used in prior studies of occupations (Burton, 1972; Coxon \& Jones, 1978; Kraus, Schild, \& Hodge, 1978), comparisons with their results are made more meaningful.

Ratings. Subjects rated each job on four scales: prestige, autonomy, concrete activity versus abstract thought, and people versus things. The scales were anchored and contained nine points. The pres- 
tige and autonomy scales were anchored at the extremes, for example, with high prestige beyond the 9 and low prestige beyond the 1 . The other two scales were bipolar, for example, concrete activity beyond the 1 , and abstract thought beyond the 9 . The scales chosen for rating were based on interpretations of previous MDS analyses of occupational title data (Burton, 1972; Coxon \& Jones, 1978). Four different random orders of job titles were constructed to reduce order effects. The scales (autonomy, prestige, etc.) were paired with each of the orderings in a balanced design (analogous to a Latin square) to reduce order effects.

\section{Analyses}

Assessing the stability of stimulus configurations. The stability of job spaces (stimulus configurations) was assessed by randomly splitting the sample into two groups. The larger group or derivation sample $(N=60)$ was used to generate a stimulus configuration; the smaller group $(N=34)$ served as a holdout. The stimulus configuration based on the derivation sample was fixed, that is, the coordinates of the points were not allowed to vary, and the holdout sample data were fit to the fixed model using ALSCAL. An $R$-square measured how well the holdout data were fit to the fixed stimulus configuration. The stability of each model was evaluated by examining shrinkage. A second way in which the stability of job spaces was assessed involved canonical correlation. Canonical correlations were computed between derivation and holdout spaces for each technique.

Comparisons were also made between techniques. The $R$-squares were compared to assess overall fit. Canonical correlation was used to assess the similarity of the spaces derived by the different unfolding models.

Ideal point comparisons across models. Rogers and Young (1981) used ALSCAL to complete successive unfolding. In this study PREFMAP (Schiffman et al., 1981) was used for the following reasons: (1) PREFMAP is more helpful in determining which of Carroll's (1972) models to use; (2) ALSCAL both normalizes and rotates the configuration when ideal points are added; if an ideal point model is used, and some subjects behave like vectors, all the stimuli and most of the ideal points will collapse into a single point on the plots, rendering the plots uninterpretable; and (3) the ALSCAL index of fit ( $R$-square) for a given individual depends on the other individuals in the analysis, which limits the meaningfulness of the fit statistic for unfolding.

\section{Results}

All internal unfoldings yielded degenerate solutions. Internal analyses were run in SAS ALSCAL for up to 90 iterations without achieving convergence. As the iterations increased, the ideal points collapsed into a single, central point, with the stimuli (jobs) spread out in a circle about the central point. Such a solution is useless for portraying individual differences. Internal unfolding was therefore eliminated from subsequent analyses.

Stimulus configurations for successive and external unfolding were generated in one through six dimensions. The final dimensionality was arrived at through consideration of fit and interpretability of the scaling solutions. The $R$-squares associated with three- and four-dimensional solutions for successive unfolding were .950 and .972 , respectively, and for external unfolding, .772 and .851 , respectively. The external $R$-squares were moderate and the successive $R$-squares were high, indicating good fit. The stress values computed by Kruskal's stress formula 1 (see Davison, 1983, p. 87), for the external stimulus configuration in three and four dimensions were .188 and .138 , respectively. Comparable stress values for the successive stimulus configurations were .118 and .083 , respectively. Dimensions beyond the third were uninterpretable for external unfolding. Consequently, three dimensions were kept for external unfolding. Successive unfolding yielded a fourth dimension that was interpretable and included in the final space.

Dimensions were labeled by inspection and by regression of the rating scales on the stimulus configuration (Kruskal \& Wish, 1978; Schiffman et al., 1981). Figures 1 and 2 show the first two di- 
Figure

First Two ALSCAL Dimensions for Successive Unfolding with Regression Labels

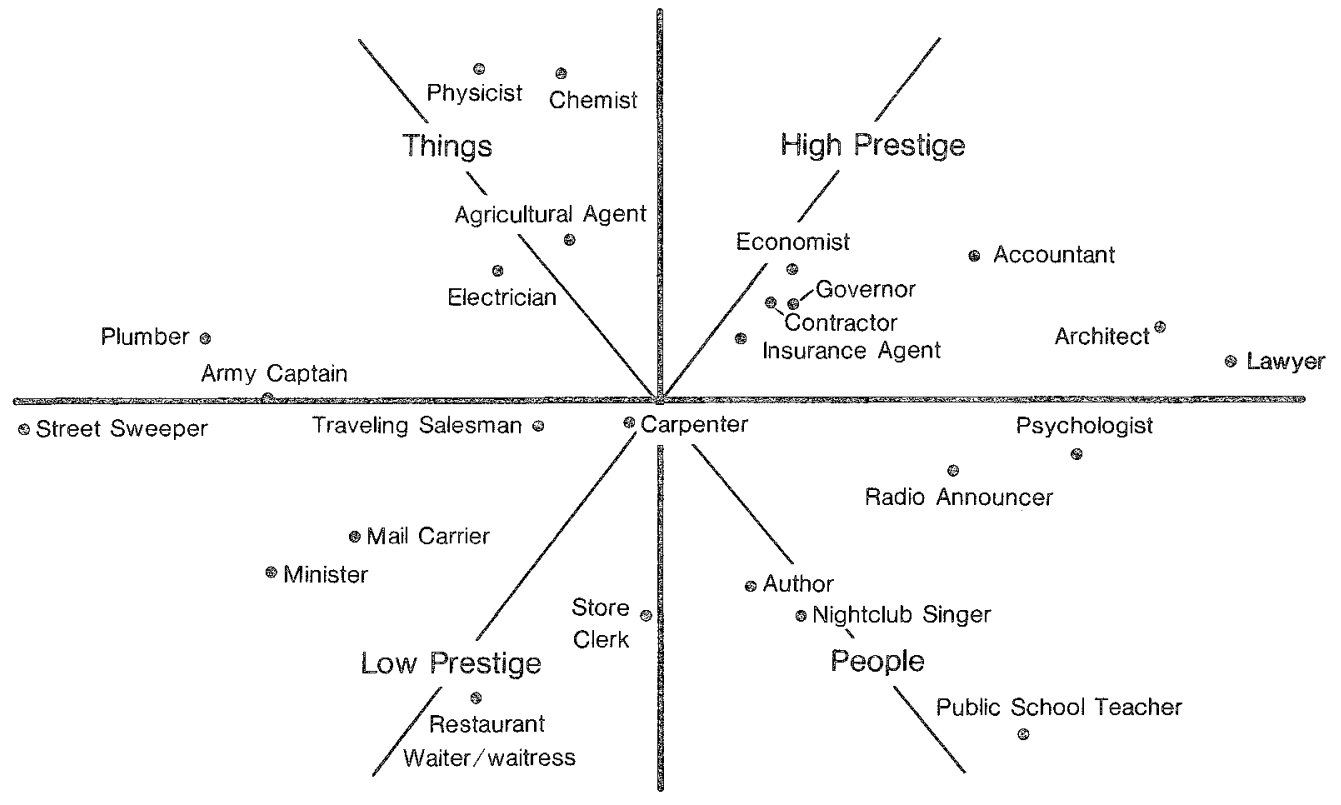

Figure 2

First Two ALSCAL Dimensions for External Unfolding with Regression Labels

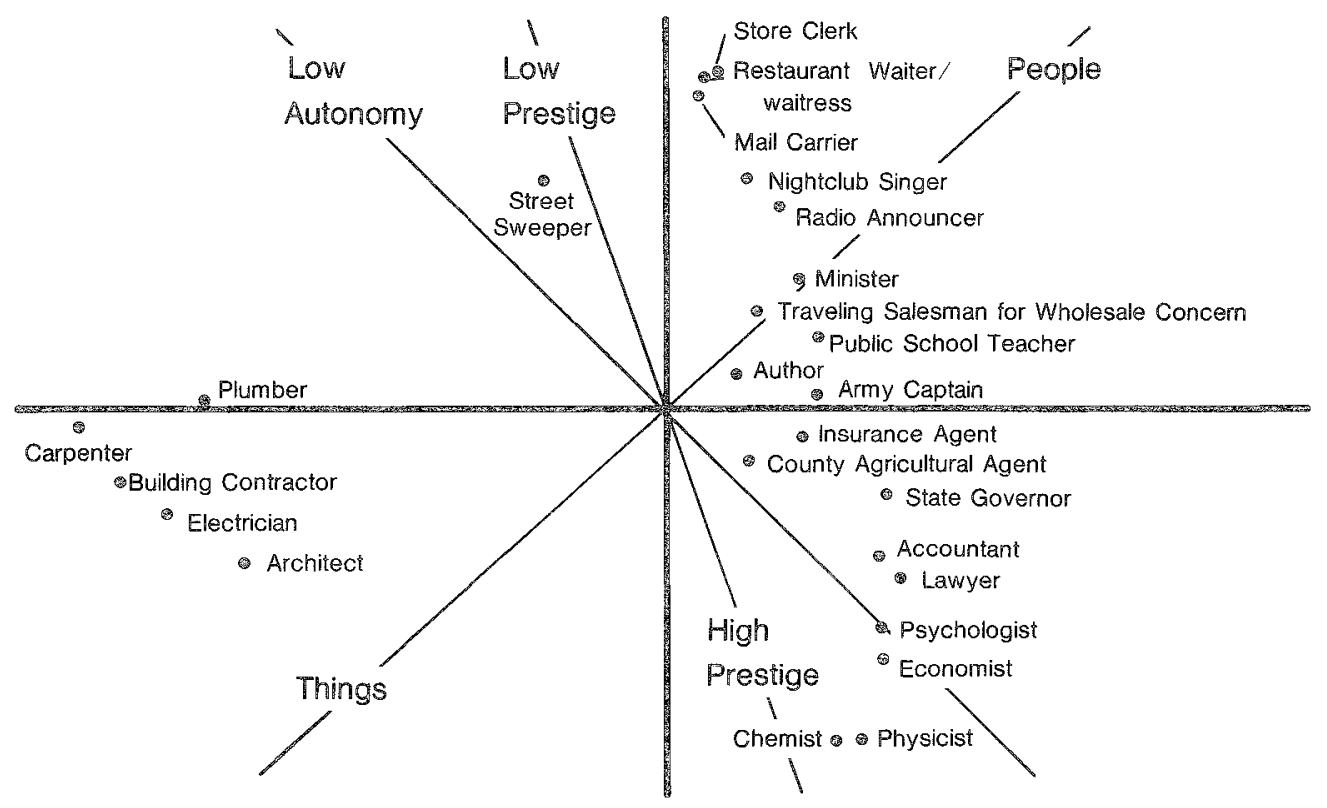

Downloaded from the Digital Conservancy at the University of Minnesota, http://purl.umn.edu/93227. May be reproduced with no cost by students and faculty for academic use. Non-academic reproduction requires payment of royalties through the Copyright Clearance Center, http://www.copyright.com/ 
mensions of the successive and external stimulus configurations, including labels from the regression analysis. The heavy horizontal and vertical axes are the original unrotated dimensions extracted by ALSCAL.

Four dimensions were uncovered using successive unfolding. The first of these was labeled prestige. Jobs at the high end of this dimension included lawyer, accountant, architect, and chemist. The low end was represented by such jobs as waiter/ waitress, store clerk, and mail carrier. The second dimension appeared to represent the distinction between people and things. The "people" jobs included public school teacher, nightclub singer, and psychologist. The "thing" jobs included physicist, chemist, and plumber. The third dimension appeared to represent a distinction between service and entertainment on one end, and conventional business on the other. Service and entertainment jobs included waiter/waitress, nightclub singer, and store clerk, whereas conventional business jobs included economist, governor, accountant, and traveling salesman. The fourth dimension represented high autonomy and thought as contrasted with low autonomy and activity. Those on the high autonomy end included author, minister, economist, and psychologist, whereas those on the activity end included carpenter, contractor, and accountant.

Three dimensions were uncovered using external unfolding. The first of these differentiates construction occupations (carpenter, contractor, electrician, plumber, etc.) from all others. The second dimension most closely resembles a prestige dimension, though autonomy is also represented. Jobs low in prestige and autonomy included store clerk, waiter/ waitress, and mail carrier. Those high in prestige and autonomy included chemist, physicist, economist, and psychologist. The third dimension reflected the distinction between thought and activity. Jobs on the thought end included chemist, physicist, author, and psychologist. Jobs on the activiry end included insurance agent, traveling salesman, and store clerk.

\section{Model Comparisons}

Several comparisons were made between successive and external unfolding solutions. Because the number of dimensions kept differed across unfolding techniques, model comparisons were carried out in both three and four dimensions. Table 1 presents the number of significant canonical variates relating the successive and external solutions. Also presented are the number of significant canonical variates relating the derivation and holdout samples for each method.

First, the stability of the stimulus space recovered by each model was assessed. The derivation $R$-squares for successive unfolding in three and four dimensions were .95 and .97 , respectively. The respective cross-validation $R$-squares were .76 and .85 , for a drop of $19 \%$ and $12 \%$ of

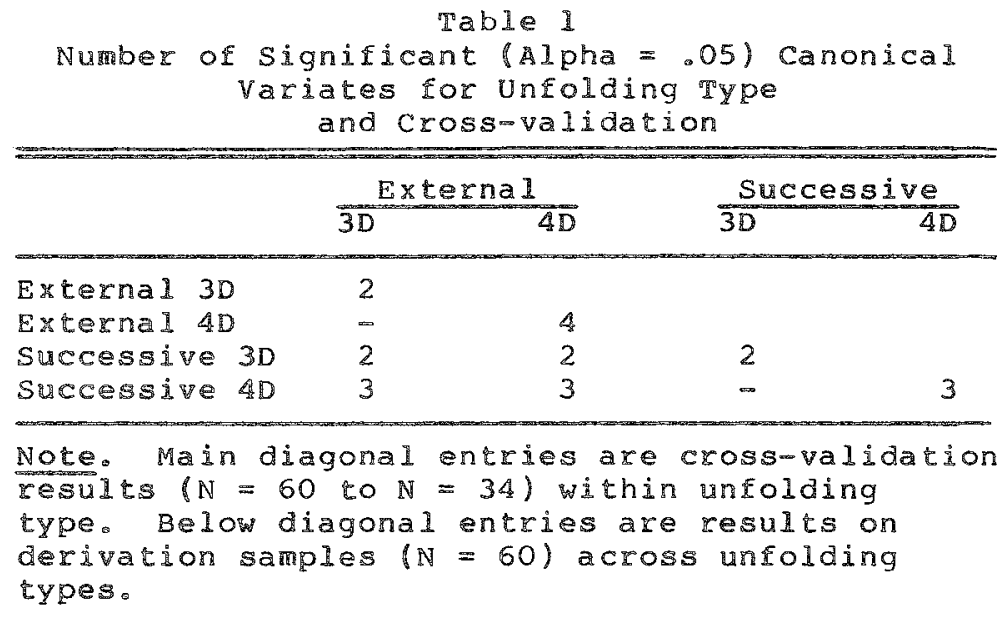

Downloaded from the Digital Conservancy at the University of Minnesota, http://purl.umn.edu/93227. May be reproduced with no cost by students and faculty for academic use. Non-academic reproduction requires payment of royalties through the Copyright Clearance Center, http://www.copyright.com/ 
the variance. Canonical correlation showed two significant variates for three dimensions, and three significant variates for four dimensions. The derivation external unfolding $R$-squares were .77 and .85 , respectively. The respective cross-validation $R$-squares were .65 and .71 , for a drop of $12 \%$ and 14\%. Canonical correlation showed two significant variates for three dimensions, and four significant variates for four dimensions. Inspection of the canonical correlation analyses suggests that much of the difference in the models' performance was due to the third dimension recovered by successive unfolding. This dimension was not evident in the cross-validation sample. For both types of unfolding, the first two dimensions were very stable in cross-validation ( $r$ greater than or equal to .90 ), and the fourth dimension was moderately stable (correlations for both models about .65).

Second, overall fit of the two models was compared. The square root of each $R$-square was transformed to Fisher's $Z$. The standard error of the difference for each comparison was computed by $(1 / 297+1 / 297)^{1 / 2}=.082$. Four comparisons were tested. The successive three- and four-dimensional derivation $R$-squares were compared to the external three- and four-dimensional derivation $R$-squares, respectively. The holdout $R$-squares were similarly compared. In each case, the successive unfolding $R$-squares were significantly larger. Successive unfolding yields a more scalable matrix in this comparison than does the external (sorting) matrix.

Table 2 contains correlations among the coordinates of the four-dimensional stimulus configurations of external and successive unfolding. The two spaces show overlap, especially for the second dimension of both spaces. The successive fourth dimension is closely related to the external third dimension. Note, however, that neither the external fourth dimension nor the successive first dimension is well predicted from the other space. Also, see Table $\mathbb{1}$ for the significant canonical variates between spaces.

To fit ideal points, PREFMAP was run with threeand four-dimensional stimulus configurations, both metrically and nonmetrically, in phases two through four (weighted ideal point to vector models). To compare fit of ideal points, (1) root-mean-square correlations for each run were examined, and (2) the number of subjects whose PREFMAP F-ratios were significant (alpha $=.05$ ) were counted. The $F$-ratios are not strictly appropriate, because the preferences are ranks. These $F$-ratios were used because (1) this technique is easily replicable, and (2) it is less arbitrary than using some value of $r$ or other measure of association whose practical meaning will change with the number of dimensions and the number of stimuli. Results are shown in Tables 3 and 4 . In all 24 comparisons, successive unfolding produced higher root-mean-square correlations and fit more people. Inspection of the between-phase $F$-ratios suggested that the vector model was the best model for a large majority of subjects in both types of unfolding.

\section{Discussion}

Rounds and Zevon (1983) reported several studies concerned with identifying dimensions of occupational perception (Burton, 1972; Coxon \& Jones, 1978, 1979a, 1979b; Kraus et al, 1978; Reeb, 1959, 1971, 1974, 1979; Shubsachs \& Davison, 1979; Siess \& Rogers, 1974). They reported that

Table 2

Correlations of Dimensions from Successive and External Derivation Stimulus spaces

\begin{tabular}{ccccc}
\hline $\begin{array}{c}\text { Successive } \\
\text { Dimensions }\end{array}$ & $\frac{\text { External }}{1}$ & Dimens ions \\
\hline 1 & .28 & -.38 & -.09 & .12 \\
2 & .02 & -.82 & .17 & .03 \\
3 & .65 & -.08 & .32 & -.01 \\
4 & .26 & .08 & .80 & .06 \\
\hline
\end{tabular}




\begin{tabular}{|c|c|c|c|c|c|c|}
\hline Met & $\begin{array}{l}\text { fic } \\
\text { cor }\end{array}$ & $\begin{array}{l}\text { QEAAP } \\
\text { Qtions } \\
\text { Fit }\end{array}$ & $\begin{array}{l}\text { Table } \\
\text { sults } \\
\text { nd No } \\
\text { er Ar }\end{array}$ & $\begin{array}{l}\text { Root } \\
\text { ber of } \\
\text { lysis }\end{array}$ & $\begin{array}{l}n-S \\
j e c\end{array}$ & \\
\hline \multirow{2}{*}{$\begin{array}{l}\text { Number of } \\
\text { Dimensions }\end{array}$} & \multirow[b]{2}{*}{$\mathrm{N}$} & \multirow[b]{2}{*}{ Phase } & \multicolumn{2}{|c|}{ Successive } & \multicolumn{2}{|c|}{ Externa } \\
\hline & & & RMS & Number & RMS & Number \\
\hline 3 & 34 & 2 & .75 & 22 & .64 & 13 \\
\hline 3 & 34 & 3 & .74 & 30 & .57 & 13 \\
\hline 3 & 34 & 4 & .72 & 32 & .55 & 15 \\
\hline 3 & 60 & 2 & .79 & 51 & .63 & 21 \\
\hline 3 & 60 & 3 & .76 & 55 & .58 & 22 \\
\hline 3 & 60 & 4 & .74 & 57 & .54 & 25 \\
\hline 4 & 34 & 2 & .82 & 26 & .71 & 10 \\
\hline 4 & 34 & 3 & .78 & 30 & .60 & 15 \\
\hline 4 & 34 & 4 & .78 & 31 & .59 & 17 \\
\hline 4 & 60 & 2 & .85 & 53 & .69 & 19 \\
\hline 4 & 60 & 3 & .81 & 57 & .60 & 21 \\
\hline 4 & 60 & 4 & .80 & 57 & .57 & 24 \\
\hline
\end{tabular}

\begin{tabular}{|c|c|c|c|c|c|c|}
\hline MOn $=\mathrm{m}$ & $\begin{array}{l}\operatorname{etr} \\
\cos \end{array}$ & $\begin{array}{c}\text { REFMAP } \\
\text { cions } \\
\text { Fit }\end{array}$ & $\begin{array}{l}\text { Resu } \\
\text { 2d } \mathrm{Nu} \\
\text { er Ar }\end{array}$ & $\begin{array}{l}\text { : ROO } \\
\text { Der of } \\
\text { YSis }\end{array}$ & jects & dare \\
\hline \multirow{2}{*}{$\begin{array}{l}\text { Number of } \\
\text { Ditmensions }\end{array}$} & \multirow[b]{2}{*}{$\mathbb{N}$} & \multirow[b]{2}{*}{ Phase } & \multicolumn{2}{|c|}{ Successive } & \multicolumn{2}{|c|}{ Excerna 1} \\
\hline & & & RMS & Number & RMS & Number \\
\hline 3 & 34 & 2 & .91 & 34 & .80 & 29 \\
\hline 3 & 34 & 3 & .88 & 34 & .74 & 28 \\
\hline 3 & 34 & 4 & .85 & 34 & .72 & 30 \\
\hline 3 & 60 & 2 & .92 & 60 & .80 & 53 \\
\hline 3 & 60 & 3 & .89 & 60 & .75 & 54 \\
\hline 3 & 60 & 4 & .86 & 60 & .72 & 54 \\
\hline 4 & 34 & 2 & .93 & 34 & .86 & 29 \\
\hline 4 & 34 & 3 & .90 & 34 & .77 & 29 \\
\hline 4 & 34 & 4 & .89 & 34 & .75 & 30 \\
\hline 4 & 60 & 2 & .94 & 60 & .85 & 53 \\
\hline 4 & 60 & 3 & .92 & 59 & .78 & 54 \\
\hline 4 & 60 & 4 & .89 & 60 & .75 & 54 \\
\hline
\end{tabular}

prestige or a very similar construct appears across all studies. Prestige was related to both types of unfolding spaces in the present study. Another dimension that is frequently but not always represented is a "blue collar versus white collar" or physical skill dimension. This dimension is seen clearly in this study only in the first dimension of the external unfolding, "construction versus oth- ers," though this dimension was moderately correlated with the successive third dimension (see Table 2). However, the successive third dimension failed to cross-validate. It is possible that the dimension of physical skill is more salient in similarity judgments, at least for this sample of students. Other dimensions vary across studies, in part because the sample of jobs used was not constant. 


\section{Model Stability}

The first two dimensions recovered by both types of unfolding were stable. In either three or four dimensions the correlation between the coordinates of the initial and cross-validation spaces was greater than or equal to .90 (after rotation). For dimensions beyond the second, external unfolding fared relatively better than successive unfolding. On the other hand, successive unfolding produced higher $R$ squares, indicating better fits. Future research might address the difference in fit found here. Might the difference be due in part to squaring the columns in calculating the proximity matrix for successive unfolding (cf. Drasgow \& Jones, 1979), or might the results have something to do with the stimuli, so that people agreed more about job preference than job classification?

\section{Heal roint Issnes}

The results of this study indicate that successive unfolding represents preference data better than external unfolding. As indicated in Tables 3 and 4, the root-mean-square correlations were higher, and more people were fit by successive unfolding than by external unfolding in every comparison. This advantage was to be expected for the derivation sample because preferences for this group enter the calculation of the stimulus configuration in successive unfolding. Note here that successive unfolding was also superior to external unfolding in representing preferences from the independent holdout sample. Since these preferences did not enter into the calculation of the stimulus space, they provide a fair comparison between the two types of unfolding. In addition, because an identical set of preferences was mapped into each unfolding model, the results cannot be attributed to one sample being more "scalable" than another. Since the difference cannot be due to the preferences, it must be due to one or more aspects of the recovered spaces.

Focusing on these spaces, the difference in fit of ideal points is not explained by differences in reliability of dimensions; external unfolding produced dimensions at least as stable across samples as those produced by successive unfolding. The difference persisted in nonmetric unfoldings, and therefore is not due to differences in the size of discrepancies between preferences and distances, but only to the number of discrepancies. Further, the difference is not due to different variances in the dimensions across unfoldings, since the difference in ideal point fit remains under a weighted unfolding model (PREFMAP phase 2).

The most reasonable interpretation of the results appears to be that the successive unfolding stimulus configuration simply did a better job of representing dimensions of preference. The first dimension of successive unfolding was a "preference" dimension related to prestige. This dimension was obviously helpful in fitting ideal points and was not strongly represented in the external unfolding solution. Table 2 shows the correlations between external and successive dimensions. Because the dimensions were extracted by ALSCAL, they are virtually orthogonal within unfolding type, and the variance of any dimension accounted for by the dimensions of the other space is approximately equal to the sum of its squared correlations. Using the data in Table 2, it can be seen that the successive first dimension is not well accounted for by the external space (about $25 \%$ common variance, as opposed to over $50 \%$ for the other dimensions, except the fourth external dimension).

The results of this study do not prove that successive unfolding is always superior to external unfolding, since the correspondence of preference and similarity based spaces will depend upon stimulus, task, and subject variables. However, successive unfolding might be the method of choice when (1) the primary interest of research is to model preferences, (2) there are a fairly large number of stimuli or time constraints, and (3) the stimuli are complex. Further investigation and application of successive unfolding appears warranted.

\section{References}

Bennett, J. F., \& Hays, W. L. (1960). Multidimensional unfolding: Determining the dimensionality of ranked preference data. Psychometrika, 25, 27-43. 
Burton, M. (1972). Semantic dimensions of occupation names. In A. K. Romney, R. N. Shepard, \& S. Nerlove (Eds.), Multidimensional scaling: Theory and applications in the behavioral sciences (Vol. II. Applications, pp. 55-71). New York: Seminar Press.

Carroll, I. D. (1972). Individual differences and multidimensional scaling. In R. N. Shepard, A. K. Romney, \& S. Nerlove (Eds.), Multidimensional scaling: Theory and applications in the behavioral sciences (Vol. I. Theory, pp. 105-155). New York: Seminar Press.

Carroll, J. D., \& Arabie, P. (1980). Multidimensional scaling. Annual Review of Psychology, 31, 607-649.

Coombs, C. H. (1950). Psychological scaling without a unit of measurement. Psychological Review, 57, 145158.

Coxon, A. P.M. (1982). The user's guide to multidimensional scaling. Exeter $\mathrm{NH}$ : Heineman.

Coxon, A. P. M., \& Jones, C. L. (1978). The images of occupational prestige. New York: St. Martin.

Coxon, A. P. M., \& Jones, C. L. (1979a). Class and hierarchy: The social meaning of occupations. New York: St. Martin.

Coxon, A. P. M., \& Jones, C. L. (1979b). Measurement and meaning: Techniques and methods of siudying occupational cognitions. New York: St. Martin.

Davison, M. L. (1976). Fitting and testing Carroll's weighted unfolding model for preferences. Psychometrika, 41, 233-247.

Davison, M. L. (1977). On a metric, unidimensional unfolding for attitudinal and developmental data. Psychometrika, 42, 523-548.

Davison, M. L. (1983). Multidimensional scaling. New York: Wiley.

Drasgow, F., \& Jones, L. E. (1979). Multidimensional scaling of derived dissimilarities. Multivariate Behavioral Research, 14, 227-244

Golledge, R. G., \& Rayner, J. N. (1982). Proximity and preference. Minneapolis: University of Minnesota Press.

Hodge, R. W., \& Siegel, P. M., \& Rossi, P. H. (1968). Occupational prestige in the United States, 1925-1963. In D. G. Zytowski (Ed.), Vocational behavior: Readings in theory and research (pp. 86-95). New York: Holt, Rinehart, \& Winston.

Kraus, V., Schild, E. O., \& Hodge, R. W. (1978). Occupational prestige in the collective conscience. Social Forces, 56, 900-918.

Kruskal, J. B., \& Wish, W. (1978). Multidimensional scaling. Beverly Hills CA: Sage Publications.

Nygren, T. E., \& Jones, L. E. (1977). Individual differences in perceptions and preferences for political candidates. Journal of Experimental Social Psychology, 13, 182-197.

Recb, M. (1959). How people sec jobs: A multidimensional analysis. Occupational Psychology, 33, 1-17.

Reeb, M. (1971). Multidimensional perceptions by counselors and 14-year-old boys of suitability for jobs, and their prestige and desirability. Occupational Psychology, 45, 233-242.

Reeb, M. (1974). The perceptions of occupational structure-an intervening variable in vocational behavior. Journal of Vocational Behavior, 4, 125-137.

Reeb, M. (1979). Occupational perception and school stream in relation to job classifications, preferences, and prestige for 17-year-old girls and boys. Journal of Occupational Psychology, 52, 113-127.

Rogers, J. L., \& Young, F. W. (1981). Successive unfolding of family preferences. Applied Psychological Measurement, 5, 51-62.

Rosenberg, S., Nelson, C., \& Vivekananthan, P. S. (1968). A multidimensional approach to the structure of personality impressions. Journal of Personality and Social Psychology, 9, 283-294.

Rounds, J. B., Jr., \& Zevon, M. A. (1983). Multidimensional scaling research in vocational psychology. Applied Psychological Measurement, 7, 491-510.

Schiffman, S. S., Reynolds, M. L., \& Young, F. W. (1981). Introduction to multidimensional scaling. New York: Academic Press.

Schubsachs, A. P. W., \& Davison, M. L. (1979). Individual differences in perceptions of occupations and occupational reinforcers. Journal of Occupational Psychology, 52, 299-310.

Siess, T. F., \& Rogers, T. B. (1974). Roe's classification and the multidimensional nature of occupational perceptions. Journal of Vocational Behavior, 4, 403 415 .

Sjöberg, L. (1980). Similarity and correlation. In E. D. Lantermann \& H. Feger (Eds.), Similarity and choice (pp. 70-87). Bern, Switzerland: Hans Huber.

Takane, Y., Young, F., \& deLeeuw, J. (1977). Nonmetric individual differences multidimensional scaling: An alternating least squares method with optimal scaling features. Psychometrika, 42, 7-67.

\section{Author's Address}

Send requests for reprints or further information to Milchael T. Brannick, Department of Psychology, Bowling Green State University, Bowling Green OH 43403, U.S.A. 